\title{
Diagnostic Value of Arginase-1 and Glypican-3 in Differential Diagnosis of Hepatocellular Carcinoma, Cholangiocarcinoma and Metastatic Carcinoma of Liver
}

\author{
Bita Geramizadeh ${ }^{1, *} ;$ Nasibe Seirfar ${ }^{2}$ \\ ${ }^{1}$ Transplant Research Center, Department of Pathology, Shiraz University of Medical Sciences, Shiraz, IR Iran \\ 2 Department of Pathology, Shiraz UNiversity of Medical Sciences, Shiraz, IR Iran \\ ${ }^{*}$ Corresponding Author: Bita Geramizadeh, Transplant Research Center, Department of Pathology, Shiraz University of Medical Sciences, Shiraz, IR Iran. Tel/Fax: +98-7136473238, \\ E-mail: geramib@gmail.com
}

Received: May 29, 2015; Accepted: June 1, 2015

\begin{abstract}
Background: Hepatocellular carcinoma is the most common primary liver cancer. Pathologic distinction between Hepatocellular Carcinoma (HCC) and adenocarcinoma (Cholangiocarcinoma (CC) and Metastatic Adenocarcinoma (MA)) can be challenging and sometimes requires immunohistochemical panels. Recently, Arginase-1 (ARG-1) and Glypican-3 (GPC-3) have been introduced for differentiation of these tumors.

Objectives: The aim of this study was to determine the diagnostic accuracy of ARG-1 and GLP-3 in differential diagnosis of liver tumors. Patients and Methods: Eighty-nine formalin-fixed paraffin-embedded tissue blocks including 43 cases of documented HCCs, 19 cases of documented CC, and 27 cases of MA involving the liver (15 colon, 5 stomach, 3 pancreas, 2 gallbladder, 1 duodenum and 1 ampulla of vater) were evaluated for immunohistochemical expression of ARG-1 and GPC-3.

Results: Arginase-1 and GPC-3 demonstrated diffuse staining, as reactivity in > 97\% of HCCs, whereas only one (5.3\%) and 2 (10.5\%) of 19 CC cases show positive staining for GPC-3 and ARG-1, respectively. The expression of both markers in MA showed 6 (22.2\%) for ARG-1 and 3 (11.1\%) for GPC-3, especially with colorectal origin. Our findings showed a statistically significant difference between ARG-1 and GPC-3 expression in HCC, CC and MA.

Conclusions: The findings of this study reveal that both ARG-1 and GPC-3 are helpful IHC markers to separate HCC from CC and MA. Furthermore, ARG-1 shows $100 \%$ sensitivity and $82.6 \%$ specificity for the diagnosis of HCC whereas GPC-3 demonstrated $97.7 \%$ sensitivity and $91.3 \%$ specificity for the diagnosis of this tumor.
\end{abstract}

Keywords: Arginase; Glypican-3; Hepatocellular Carcinoma; Cholangiocarcinoma; Adenocarcinoma

\section{Background}

Hepatocellular Carcinoma (HCC) is the most common primary liver cancer. It is the fifth common cancer worldwide and the third leading cause of cancer-related death, after lung and stomach cancers (1).

The distinction of HCC from cholangiocarcinoma (CC) and other types of adenocarcinoma metastatic to the liver is very important. However, in most cases; the correct diagnosis can be made by the combination of clinical findings, imaging modalities and routine evaluation of Hematoxylin and Eosin (H \& E) stained sections.

Immunohistochemistry plays a very crucial role in differential diagnosis of liver tumors (2). There are some immunohistochemical markers for identification of hepatocyte origin in routine surgical pathology practice, such as Hepatocyte Paraffin Antigen-1 (HepPar-1), polyclonal Carcinoembryonic Antigen (CEA), CD10, and Alpha-Fetoprotein (AFP) (3). However, the sensitivity and specificity of these markers are relatively low, for example the sensitivity of AFP ranges from 30\% to 50\% with frequent focal staining. Polyclonal CEA and CD10 can be difficult to interpret because of diffuse cytoplasmic and canalicular staining (4). Also, the sensitivities of these markers can be low (25\% to 50\%) in poorly differentiated HCCs (5). Meanwhile, HepPar-1 can be negative in small Tru-cut needle biopsies or variants of HCC such as clear cell type (3).

Recent literature report characterized new immunohistochemical markers, Arginase-1 (ARG-1) and GLP-3 as potential markers of hepatocellular differentiation in both surgical pathology and cytopathology that may at last prove to be useful diagnostic tools in surgical pathology practice by increasing sensitivity and specificity of previous markers. However, there are not so many studies about their task.

\section{Objectives}

The purpose of this study was to investigate and de-

Copyright ( 2015, Kowsar Corp. This is an open-access article distributed under the terms of the Creative Commons Attribution-NonCommercial 4.0 International License (http://creativecommons.org/licenses/by-nc/4.0/) which permits copy and redistribute the material just in noncommercial usages, provided the original work is properly cited. 
Geramizadeh B et al.

scribe ARG-1 and GLP-3 immunostaining utility in differential diagnosis of HCC, CC and metastatic liver tumors.

\section{Patients and Methods}

This study consisted of 43 documented cases of HCC, 27 cases of metastatic carcinoma to the liver (15 colon, 5 stomach, 3 pancreas, 2 gallbladder, 1 duodenum and 1 ampulla of vater), and 19 cases of CC. All cases were retrieved from the archives of the pathology department, Shiraz University of Medical Sciences during the period between 2010 and 2014. The clinical history, pathology reports and $\mathrm{H} \& \mathrm{E}$ stained slides for all cases were reviewed to confirm the diagnosis. The histologic diagnosis of HCC was made on surgically resected $(n=67)$ and needle biopsy $(\mathrm{n}=22$ ) specimens. Hepatocellular carcinomas were classified as well-differentiated $(n=40)$, moderately differentiated $(n=1)$, or poorly differentiated $(n=1)$, respectively, corresponding to World Health Organization criteria (1). Cases of CC were characterized histologically by proliferating glands or tubules with an associated fibrous stroma in the explanted livers, and were confirmed by clinical exclusion of an extrahepatic primary tumor. In all cases of MA, the primary site was well-established histologically by a previously resected extrahepatic primary tumor and/or a clinical history of a known primary tumor outside of the liver.

Demographic findings of the cases are shown in Table 1.

The cases were all reviewed by two pathologists and confirmed the previous diagnosis, and then the best slide of the tumor was selected and the corresponding paraffin block for immunohistochemistry was isolated.

The antibodies were rabbit polyclonal antibody against ARG-1 (H-52: sc 20150, Santa Cruz, Europe) at a dilution 1:500, and monoclonal mouse, anti-GLP-3 antibody concentrate (Cell Marque, USA), clone 1G12.

Normal adult liver tissue staining was considered as positive internal controls for ARG-1 (1). GLP-3 has no reactivity in normal adult liver but is positive in fetal liver, so fetal liver from autopsy cases was used as a positive con$\operatorname{trol}(6)$.

The stained slides were assessed independently by two surgical pathologists. Only cytoplasmic and /or nuclear reactivity for ARG-1 and cytoplasmic and/or membranous reactivity for GLP-3 was considered as positive staining. We evaluated staining intensity and percentage of the tumor cells stained. The staining was scored as 0 (negative), $1+$ (weak), $2+$ (moderate) and $3+$ (strong). The number of positive tumor cells was recorded as focal $(<10 \%)$, patchy $(10 \%-50 \%)$ or diffuse (>50\%). Then the result of the immunohistochemistry of the two markers (ARG-1 and GLP-3) compared with the final diagnosis $(1,6)$.

Statistical analysis was performed by SPSS version 16 for windows. Group comparisons of categorical variables were analyzed using the Pearson's chi-square test. Finally, sensitivity, specificity, Positive Predictive Value (PPV), and Negative Predictive Value (NPV) of ARG-1 and GLP-3 were calculated. All P values that were two-sided and less than 0.05 were considered as statistically significant, and less than 0.01 were considered highly significant.

\section{Results}

Strong and diffuse cytoplasmic and nuclear staining for ARG-1 was observed in all 43 HCCs. (Figures $1 \mathrm{~A}$ and 1B) GLP-3 immunostaining was diffusely cytoplasmic and/ or membranous positive in 42 of 43 (97.7\%) cases of HCC (Figure 1 C) whereas one of 40 (2.5\%) cases of the well- differentiated HCC was negative. In our study, staining intensity of ARG-1 and GLP-3 were at least moderate (2+) staining in the majority of the cases of HCC.

Arginase- 1 and GLP-3 staining was negative in 21 (77.8\%) and 24 (88.9\%) of 27 MA cases. Arginase-1 demonstrated immunoreactivity in 6 (22.2\%) cases of adenocarcinoma, especially colorectal carcinoma, which showed weak staining in 4 of $15(26.7 \%)$ cases. Also, one of 5 (20\%) gastric adenocarcinoma cases presented $2+$ intensity and diffuse staining for ARG-1. Glypican was positive in 3 (11.1\%) of adenocarcinoma, seen in 2 colorectal and one gastric adenocarcinoma (Figure $2 \mathrm{D}$ ).

All cases of MA with primary origin of pancreas, gallbladder and ampulla of vater showed no immunoreactivity for two markers; however, few cases of colorectal cancers showed weak positivity. Glypican-3 and ARG-1 were negative in $94.7 \%$ and $89.5 \%$ of CC, respectively (Figures $3 \mathrm{~A}, 3 \mathrm{~B}$ and $3 \mathrm{C}$ ). Table 2 shows the summary of the above findings.

Table 1. Demographic Findings of the Patients in Three Groups of the Specimens ${ }^{\mathrm{a}}$

\begin{tabular}{lccc}
\hline & Cases & Age, $\mathbf{y}$ & Male \\
\hline Hepatocellular carcinoma & $43(48.3)$ & $43(1-81)$ & 59 \\
Metastasis & $27(30.4)$ & $53(2-80)$ & 18 \\
Cholangiocarcinoma & $19(21.3)$ & $49(21-80)$ & 9 \\
Total & $89(100)$ & $47(1-81)$ & 59 \\
\hline
\end{tabular}

a Data are presented as mean (range) or No. (\%). 


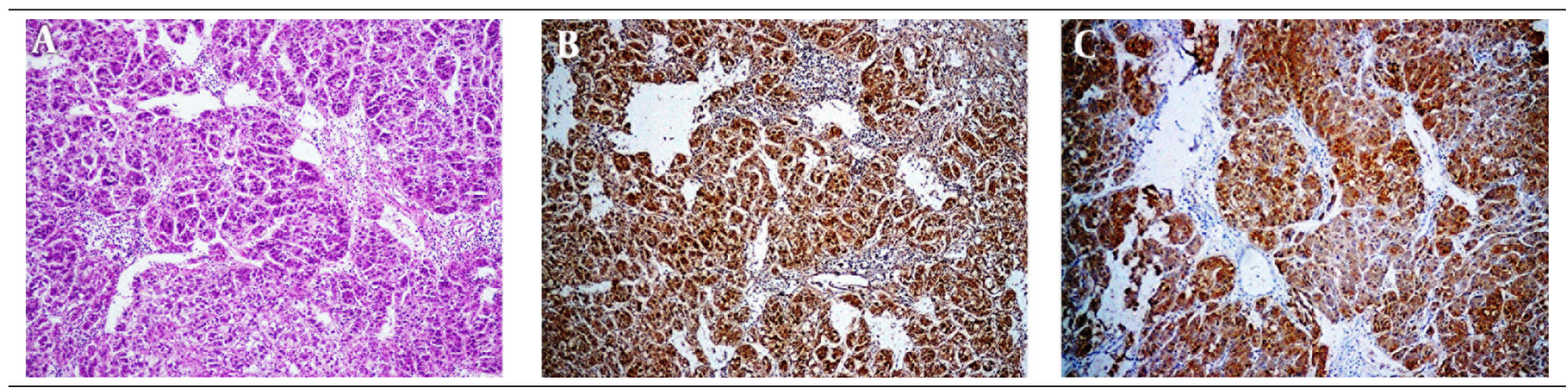

Figure 1. A, Sections Show Typical HCC (H \& E Stain x100); B, Arginase Stain Shows Positive Cytoplasm; C, Glypican Shows Positive Cytoplasm
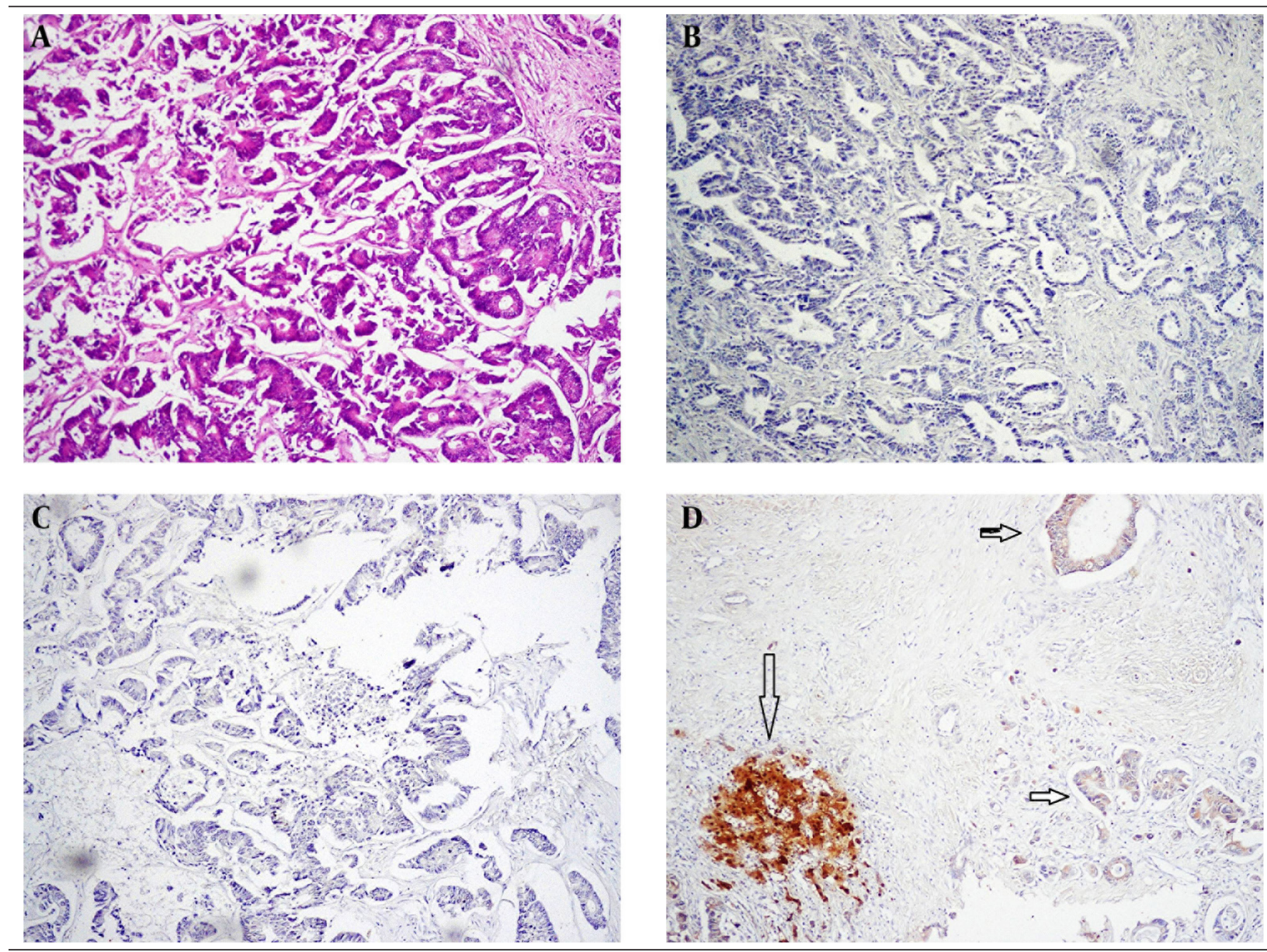

Figure 2. A, Sections Show Metastatic Adenocarcinoma From Colon (H \& E x100); B, Arginase Staining Shows Negative Cytoplasm; C, Glypican Shows Negative Cytoplasm; D, A Case of Metastatic Adenocarcinoma of Colon Which Shows Weak Positivity of the Metastatic Glands, Horizontal Arrow (Compare With Intense Staining With the Normal Liver, Vertical Arrow)
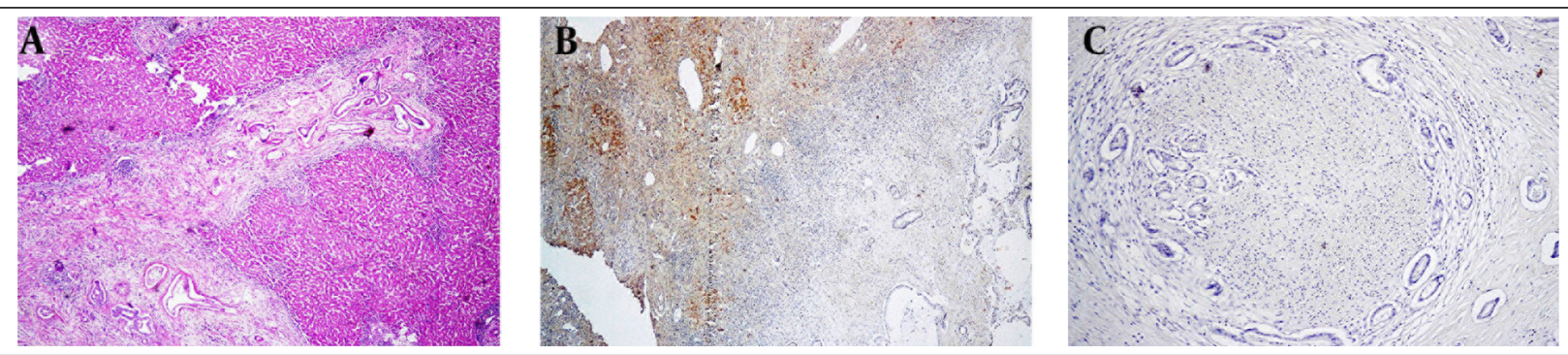

Figure 3. A, Sections From Cholangiocarcinoma (H \& Ex100); B, Arginase Staining Shows Negative Cytoplasm (Note the Intense Staining of the Normal Liver (Vertical Arrow) in the Vicinity of Negative Glands (Horizontal Arrow); C, Glypican Shows Negative Cytoplasm 
Geramizadeh B et al.

\begin{tabular}{|c|c|c|c|c|c|c|c|c|c|c|c|}
\hline \multirow[t]{3}{*}{ Effects } & \multirow[t]{3}{*}{ Cases } & \multicolumn{5}{|c|}{ Arginase-1 } & \multicolumn{5}{|c|}{ Glypican-3 } \\
\hline & & \multicolumn{2}{|l|}{ Negative } & \multicolumn{2}{|l|}{ Positive } & \multirow{2}{*}{$\begin{array}{c}\begin{array}{c}\text { Total } \\
\text { Positive }\end{array} \\
\text { No.(\%) }\end{array}$} & \multirow{2}{*}{$\begin{array}{c}\text { Negative } \\
\mathbf{0}(0 \%)\end{array}$} & \multicolumn{3}{|c|}{ Positive } & \multirow{2}{*}{$\begin{array}{c}\begin{array}{c}\text { Total } \\
\text { Positive }\end{array} \\
\text { No. (\%) }\end{array}$} \\
\hline & & $\mathbf{0}(\mathbf{0} \%)$ & $1+(\%)$ & $2+(\%)$ & $3+(\%)$ & & & $\mathbf{1}+(\%)$ & $2+(\%)$ & $3+(\%)$ & \\
\hline Hepatocellular carcinma & 43 & $0(0)$ & $4(9.3)$ & $25(58.1)$ & $14(35)$ & $43(100)$ & $1(2.3)$ & $3(7)$ & $29(67.4)$ & $10(23.3)$ & $42(97.7)$ \\
\hline Well- differentiated & 40 & $0(0)$ & $4(10)$ & $22(55)$ & $0(0)$ & $40(100)$ & $1(2.5)$ & $3(7.5)$ & $26(65)$ & $10(25)$ & $39(97.5)$ \\
\hline Moderately differentiated & 1 & $0(0)$ & $0(0)$ & $1(100)$ & $0(0)$ & $1(100)$ & $0(0)$ & $0(0)$ & $1(100)$ & $0(0)$ & $1(100)$ \\
\hline Poorly differentiated & 1 & $0(0)$ & $0(0)$ & $1(100)$ & $0(0)$ & $1(100)$ & $0(0)$ & $0(0)$ & $1(100)$ & $0(0)$ & $1(100)$ \\
\hline Fibrolamellar & 1 & $0(0)$ & $0(0)$ & $1(100)$ & $0(0)$ & $1(100)$ & $0(0)$ & $0(0)$ & $1(100)$ & $0(0)$ & $1(100)$ \\
\hline Metastatic carcinma & 27 & $21(77.8)$ & $5(18.5)$ & $1(3.7)$ & $0(0)$ & $6(22.2)$ & $24(88.9)$ & $2(7.4)$ & $1(3.7)$ & $0(0)$ & $3(11.1)$ \\
\hline Colonic & 15 & $11(73.3)$ & $4(26.7)$ & $0(0)$ & $0(0)$ & $4(26.7)$ & $13(86.7)$ & $2(13.3)$ & $0(0)$ & $0(0)$ & $2(13.3)$ \\
\hline Gastric & 5 & $4(80)$ & $0(0)$ & $1(20)$ & $0(0)$ & $1(20)$ & $4(80)$ & $0(0)$ & $1(20)$ & $0(0)$ & $1(20)$ \\
\hline Pancreas & 3 & $3(100)$ & $0(0)$ & $0(0)$ & $0(0)$ & $0(0)$ & $3(100)$ & $0(0)$ & $0(0)$ & $0(0)$ & $0(0)$ \\
\hline Gallbladder & 2 & $2(100)$ & $0(0)$ & $0(0)$ & $0(0)$ & $0(0)$ & $2(100)$ & $0(0)$ & $0(0)$ & $0(0)$ & $0(0)$ \\
\hline Duodenum & 1 & $0(0)$ & $1(100)$ & $0(0)$ & $0(0)$ & $1(100)$ & $1(100)$ & $0(0)$ & $0(0)$ & $0(0)$ & $0(0)$ \\
\hline Ampulla of vater & 1 & $1(100)$ & $0(0)$ & $0(0)$ & $0(0)$ & $0(0)$ & $1(100)$ & $0(0)$ & $0(0)$ & $0(0)$ & $0(0)$ \\
\hline Cholangiocarcinma & 19 & $17(89.5)$ & $2(10.5)$ & $0(0)$ & $0(0)$ & $2(10.5)$ & $18(94.7)$ & $1(5.3)$ & $0(0)$ & $0(0)$ & $1(5.3)$ \\
\hline
\end{tabular}

\begin{tabular}{lcccc}
\hline \multicolumn{5}{l}{ Table 3. Sensitivity, Specificity, Positive and Negative Predictive Value of Arginase-1 and Glypican-3 for HCC Diagnosis a,b } \\
\hline & Sensitivity & Specificity & PPV & NPV \\
\hline ARG-1 & 100 & 82.6 & 84.3 & 100 \\
GLP-3 & 97.7 & 91.3 & 91.3 & 97.7 \\
\hline
\end{tabular}

a Abbreviations: ARG-1, arginase-1; GLP-3, glypican-3; PPV, positive predictive value; NPV, negative predictive value.

b Data are presented as \%.

It is worthy to note that none of the cases of CC and MA showed intense and more than 2+ reactivity with ARG-1 and GLP-3.

Sensitivity, specificity, positive and negative predictive values of ARG-1 and GLP-3 in distinguishing HCC from MA and CC were offered as follows: $100 \%, 82.6 \%, 84.3 \%, 100 \%$, for ARG1and 97.7\%, 91.3\%, 91.3\%, 97.7\% for GLP-3, respectively (Table 3).

\section{Discussion}

Pathological distinction between different types of liver tumors, mainly composed of HCC, CC and MA, can be very challenging, particularly in core needle biopsies (3).

There are some immunohistochemical markers for HCC including Hepatocyte Specific Antigen (HAS or Hep Par1), Alpha-Fetoprotein (AFP), Carcinoembronic Antigen (CEA), CD10, and CD34. Unfortunately the sensitivity and specificity of these markers are low and have significant diagnostic limitations (7).
Arginase-1 and GLP-3 are new immunohistochemical markers that have been reported to be expressed in HCC (2). Therefore, primary purpose of the present study was to examine the IHC study of ARG-1 and GLP-3 in documented cases of HCC, MA, and CC.

There are quite a few studies regarding the importance of ARG-1 immunostaining in the diagnosis of HCC. Table 4 summarized the result of published studies on ARG-1 and GLP-3 expression in HCC. The percentage of ARG-1 expression reported in this tumor has ranged from $81 \%$ to $96 \%$ of the cases (Table 4). In our study ARG-1 positivity was demonstrated in 43 (100\%) of 43 HCC, which were higher than those reported in some of the previous studies. In our study, GLP-3 has also been expressed in 42 (97.7\%) of HCCs, this is nearly similar to the previous studies (8). This finding is in accordance with previous observation, which indicated positive rate of GLP-3 ranging between $49 \%$ and $97 \%$ in HCC (Table 4 ). 
Geramizadeh B et al.

Among nonhepatocellular tumors, ARG-1 was negative in $21(77.8 \%)$ of 27 adenocarcinomas. This finding shows statistically significant difference between ARG-1 expression in HCC and MA cases. Fujiwara et al. demonstrated immunoreactivity for ARG-1 in 6 (10\%) of 61 total adenocarcinoma cases by using fine-needle aspiration material, which showed immunoreactivity can be identified in adenocarcinomas, particularly in pancreatic origin (19). In the study by Radwan and Ahmed (1) in one case of pancreatic adenocarcinoma of 38 (2.6\%) MA cases, ARG-1 has been positive (1). Although the number of available studies on the expres- sion of GLP-3 in nonhepatocellular tumors whole sections is limited, in the literature GLP-3 positivity in MA cases ranged from 0 (0\%) to 16.7\% in FNA material (8,17-19). Glypican-3 was detected in 3 of the 50 MA cases in the study of Yan et al. on whole sections (16). In our data, 24 (88.9\%) of 27 MA cases were negative for GLP-3 similar to the study by Zaakook et al.(9) who found that GPC-3 was negative in $83.3 \%$ of MA cases. Expression in CC has been described but is rare. In our data only one (5.3\%) and 2 (10.5\%) of 19 CC cases have positive staining for GLP-3 and ARG-1 respectively, which this result confirms other investigations (Tables 5 and 6).

Table 4. Previous Studies on Arginase-1 and Glypican-3 Expression in HCC in Comparison With the Current Study a,b

\begin{tabular}{|c|c|c|c|c|c|}
\hline \multirow[t]{2}{*}{ Studies } & \multicolumn{2}{|c|}{ Arginase-1 } & \multicolumn{2}{|c|}{ Glypican-3 } & \multirow[t]{2}{*}{ Type of Specimen } \\
\hline & $\mathbf{n}$ & Positive Cases & $\mathbf{n}$ & Positive Cases & \\
\hline Radwan and Ahmed (1) & 50 & $42(84)$ & - & - & Whole sections \\
\hline Zaakook et al. (9) & - & - & 42 & $40(95.2)$ & FNA cell block \\
\hline Yan et al. (10) & - & - & 757 & $492(65)$ & Whole sections \\
\hline Anatelli et al. (11) & - & - & 74 & $36(49)$ & Whole sections \\
\hline Kring et al. (12) & 160 & $151(94)$ & 172 & $121(70)$ & Whole sections \\
\hline Shirakawa et al. (13) & - & - & 46 & $36(78)$ & Whole sections \\
\hline Shafizadeh et al. (14) & - & - & 58 & $46(79)$ & Whole sections \\
\hline Wang et al. (7) & - & - & 111 & $84(75.7)$ & Whole sections \\
\hline Wang et al. (15) & - & - & 54 & $38(70)$ & Tissue microarray \\
\hline Yamauchi et al. (6) & - & - & 56 & $47(84)$ & Whole sections \\
\hline Sang et al. (2) & 78 & $75(96.1)$ & - & - & Whole sections \\
\hline Yan et al. (16) & 151 & $145(96)$ & - & - & Tissue microarray \\
\hline McKnight et al. (17) & 44 & $37(84)$ & 44 & $25(57)$ & FNA cell block \\
\hline Timek et al. (18) & 29 & $23(79)$ & 29 & $25(86)$ & FNA cell block \\
\hline Fujiwara et al. (19) & 37 & $30(81)$ & 37 & $20(54)$ & FNA cell block \\
\hline Ibrahim et al. (8) & - & - & 30 & $29(97)$ & FNA cell block \\
\hline Current study & 43 & $43(100)$ & 43 & $42(97.7)$ & Whole sections \\
\hline
\end{tabular}

a Abbreviations: FNA, Fine-needle aspiration.

b Data are presented as No. (\%).

Table 5. Shows the Previous Studies on Arginase-1 and Glypican-3 Expression in Nonhepatocellular Tumors (Metastatic AC) ${ }^{a}$

\begin{tabular}{|c|c|c|c|c|c|}
\hline \multirow[t]{2}{*}{ Studies } & \multicolumn{2}{|c|}{ Arginase-1 } & \multicolumn{2}{|c|}{ Glypican-3 } & \multirow[t]{2}{*}{ Type of Specimen } \\
\hline & $\mathbf{n}$ & Positive Cases & $\mathbf{n}$ & Positive Cases & \\
\hline $\begin{array}{l}\text { Radwan and Ahmed } \\
\text { (1) }\end{array}$ & 38 & $1(2.6)$ & - & - & Whole sections \\
\hline Zaakook et al. (9) & - & - & 18 & $3(16.7)$ & FNA cell block \\
\hline Yan et al. (10) & - & - & 50 & $3(6)$ & Whole sections \\
\hline Yamauchi et al. (6) & - & - & 23 & $1(4.34)$ & Whole sections \\
\hline Sang et al. (2) & 34 & $0(0)$ & & & Whole sections \\
\hline Yan et al. (16) & 99 & $0(0)$ & - & - & Tissue microarray \\
\hline McKnight et al. (17) & 35 & $0(0)$ & 35 & $0(0)$ & FNA cell block \\
\hline Timek et al. (18) & 28 & $0(0)$ & 28 & $3(10.7)$ & FNA cell block \\
\hline Fujiwara et al. (19) & 61 & $6(10)$ & 61 & $5(8)$ & FNA cell block \\
\hline Ibrahim et al. (8) & - & - & 18 & $0(0)$ & FNA cell block \\
\hline Current study & 27 & $6(22.2)$ & 27 & $3(11.1)$ & Whole section \\
\hline
\end{tabular}

${ }^{\mathrm{a}}$ Data are presented as No.(\%). 
Table 6. Previous Studies on Arginase-1 and Glypican-3 Expression in Nonhepatocellular Tumors (CC) in Comparison to the Current Study a

\begin{tabular}{|c|c|c|c|c|c|}
\hline \multirow[t]{2}{*}{ Studies } & \multicolumn{2}{|c|}{ Arginase-1 } & \multicolumn{2}{|c|}{ Glypican-3 } & \multirow[t]{2}{*}{ Type of Specimen } \\
\hline & $\mathbf{n}$ & Positive Cases & $\mathbf{n}$ & Positive Cases & \\
\hline Radwan and Ahmed (1) & 12 & $1(8.3)$ & - & - & Whole sections \\
\hline Yan et al. (10) & - & - & 99 & $2(2.3)$ & Whole sections \\
\hline Kring et al. (12) & 16 & $0(0)$ & 16 & $1(6)$ & Whole sections \\
\hline Shirakawa et al. (13) & - & - & 28 & $0(0)$ & Whole sections \\
\hline Wang et al. (7) & - & - & 10 & $1(10)$ & Tissue microarray \\
\hline Yamauchi et al. (6) & - & - & 16 & $0(0)$ & Whole sections \\
\hline Yan et al. (16) & 6 & $1(16.6)$ & - & - & Tissue microarray \\
\hline Timek et al. (18) & 11 & $0(0)$ & 11 & $0(0)$ & FNA cell block \\
\hline Current study & 19 & $2(10.5)$ & 19 & $1(5.3)$ & Whole sections \\
\hline
\end{tabular}

a Data are presented as No.(\%).

In our study, the sensitivity of GPC-3 in diagnosing HCC was $97.7 \%$, the specificity $91.3 \%$, PPV 91.3\% and NPV 97.7\%. These findings are similar to the findings of Ibrahim et al. (8) which in their study, the sensitivity of GPC-3 in HCC was $96.7 \%$, specificity was $100 \%$, PPV $100 \%$ and NPV $94.7 \%$. In the study of Timek et al. (18) the sensitivity of GPC-3 was $83 \%$, specificity $96.7 \%$, but Fujiwara et al. (19) showed lower sensitivity of GPC-3 which was $54 \%$, specificity $92 \%$, PPV 80\% and NPV 77\%.

In this study the sensitivity of ARG-1 in diagnosing HCC was $100 \%$, the specificity $82 \%$, PPV $84.3 \%$ and NPV of $100 \%$. These findings are nearly similar to the findings of the previous studies. Sang et al. (2) reported that the sensitivity was $96.1 \%$, specificity 99.6\%, PPV 98.7\% and NPV 98.8\%. McKnight et al. (17) found that sensitivity of ARG-1 in distinguishing HCC from other malignant non-HCC lesions was $84.1 \%$, specificity $92.2 \%$, PPV $74 \%$ and NPV $83.3 \%$. Radwan and Ahmed (1) found that sensitivity was $84 \%$, the specificity $96 \%$, PPV $95.5 \%$ and NPV $85.7 \%$. In the study of Fujiwara et al. (19) the sensitivity of ARG-1 was $81 \%$, specificity $90 \%$, PPV $83 \%$ and NPV $89 \%$.

In conclusion, our results show a high frequency of positive staining for ARG-1 and GLP-3 in HCC that these are useful diagnostic immunomarkers to distinguish HCC from CC and MA.

\section{Acknowledgements}

We wish to express our deep appreciation to Mrs. Tahere Heidari and Marjan Rahsaz for their kind cooperation in preparing IHC slides and we also would like to thank the staff of pathology department of Namazi Hospital for preparing excellent pathology sections.

\section{Authors' Contributions}

Bita Geramizadeh: Design of the project, reading the slides, and writing the paper. Nasibe Seirfar: preparation and reading the slides.

\section{Funding/Support}

This paper was prepared according to the project No5088-91 of Shiraz University of Medical Sciences, Shiraz, IR Iran.

\section{References}

1. Radwan NA, Ahmed NS. The diagnostic value of arginase-1 immunostaining in differentiating hepatocellular carcinoma from metastatic carcinoma and cholangiocarcinoma as compared to HepPar-1. Diagn Pathol. 2012;7:149.

2. Sang W, Zhang W, Cui W, Li X, Abulajiang G, Li Q. Arginase-1 is a more sensitive marker than HepPar-1 and AFP in differential diagnosis of hepatocellular carcinoma from nonhepatocellular carcinoma. Tumor Biology. 2015;36(5):3881-6.

3. Geramizadeh B, Boub R, Rahsaz M. Histologic differentiation of hepatocellular carcinoma from adenocarcinoma by a simple panel: evaluation of the pitfalls. Indian J Pathol Microbiol. 2007;50(3):507-10.

4. Gomaa AI, Khan SA, Toledano MB, Waked I, Taylor-Robinson SD. Hepatocellular carcinoma: epidemiology, risk factors and pathogenesis. World J Gastroenterol. 2008;14(27):4300-8.

5. Satir AA. An Update on the Pathogenesis and Pathology of Hepatocellular Carcinoma. Bahrain Med Bull. 2007;29(2):1-8.

6. Yamauchi N, Watanabe A, Hishinuma M, Ohashi K, Midorikawa Y, Morishita Y, et al. The glypican 3 oncofetal protein is a promising diagnostic marker for hepatocellular carcinoma. Mod Pathol. 2005;18(12):1591-8.

7. Wang HL, Anatelli F, Zhai QJ, Adley B, Chuang ST, Yang XJ. Glypican-3 as a useful diagnostic marker that distinguishes hepatocellular carcinoma from benign hepatocellular mass lesions. Arch Pathol Lab Med. 2008;132(11):1723-8.

8. Ibrahim TR, Abdel-Raouf SM. Immunohistochemical Study of Glypican-3 and HepPar-1 in Differentiating Hepatocellular Carcinoma from Metastatic Carcinomas in FNA of the Liver. Pathol Oncol Res. 2015;21(2):379-87.

9. Zaakook M, Ayoub M, Sinna EA, El-Sheikh S. Role of glypican-3 immunocytochemistry in differentiating hepatocellular carcinoma from metastatic carcinoma of the liver utilizing fine needle aspiration cytology. J Egypt Natl Canc Inst. 2013;25(4):173-80.

10. Yan B, Wei JJ, Qian YM, Zhao XL, Zhang WW, Xu AM, et al. Expression and clinicopathologic significance of glypican 3 in hepatocellular carcinoma. Ann Diagn Pathol. 2011;15(3):162-9.

11. Anatelli F, Chuang ST, Yang XJ, Wang HL. Value of glypican 3 immunostaining in the diagnosis of hepatocellular carcinoma on needle biopsy. Am J Clin Pathol. 2008;130(2):219-23.

12. Krings G, Ramachandran R, Jain D, Wu TT, Yeh MM, Torbenson M, 
et al. Immunohistochemical pitfalls and the importance of glypican 3 and arginase in the diagnosis of scirrhous hepatocellular carcinoma. Mod Pathol. 2013;26(6):782-91.

13. Shirakawa H, Kuronuma T, Nishimura Y, Hasebe T, Nakano M, Gotohda N, et al. Glypican-3 is a useful diagnostic marker for a component of hepatocellular carcinoma in human liver cancer. Int J Oncol. 2009;34(3):649-56.

14. Shafizadeh N, Ferrell LD, Kakar S. Utility and limitations of glypican-3 expression for the diagnosis of hepatocellular carcinoma at both ends of the differentiation spectrum. Mod Pathol. 2008;21(8):1011-8.

15. Wang XY, Degos F, Dubois S, Tessiore S, Allegretta M, Guttmann $\mathrm{RD}$, et al. Glypican-3 expression in hepatocellular tumors: diagnostic value for preneoplastic lesions and hepatocellular carcinomas. Hum Pathol. 2006;37(11):1435-41.
16. Yan BC, Gong C, Song J, Krausz T, Tretiakova M, Hyjek E, et al. Arginase-1: a new immunohistochemical marker of hepatocytes and hepatocellular neoplasms. Am J Surg Pathol. 2010;34(8):1147-54.

17. McKnight R, Nassar A, Cohen C, Siddiqui MT. Arginase-1: a novel immunohistochemical marker of hepatocellular differentiation in fine needle aspiration cytology. Cancer Cytopathol. 2012;120(4):223-9.

18. Timek DT, Shi J, Liu H, Lin F. Arginase-1, HepPar-1, and Glypican-3 are the most effective panel of markers in distinguishing hepatocellular carcinoma from metastatic tumor on fine-needle aspiration specimens. Am J Clin Pathol. 2012;138(2):203-10.

19. Fujiwara M, Kwok S, Yano H, Pai RK. Arginase-1 is a more sensitive marker of hepatic differentiation than HepPar-1 and glypican-3 in fine-needle aspiration biopsies. Cancer Cytopathol. 2012;120(4):230-7. 